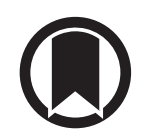

CrossMark

\title{
Making the case for causality: what role do lung microbiota play in idiopathic pulmonary fibrosis?
}

\author{
Robert P. Dickson ${ }^{1,2,3}$, Sergio Harari $\mathbb{1}^{4}$ and Martin Kolb $\mathbb{1}^{5}$ \\ Affiliations: ${ }^{1}$ Division of Pulmonary and Critical Care Medicine, Dept of Internal Medicine, University of \\ Michigan Medical School, Ann Arbor, MI, USA. ${ }^{2}$ Dept of Microbiology and Immunology, University of Michigan \\ Medical School, Ann Arbor, MI, USA. ${ }^{3}$ Michigan Center for Integrative Research in Critical Care, Ann Arbor, \\ MI, USA. ${ }^{4}$ Dept of Medical Sciences San Giuseppe Hospital MultiMedica IRCCS and Dept of Clinical Sciences \\ and Community Health, University of Milan, Milan, Italy. ${ }^{5}$ McMaster University and St. Joseph's Healthcare, \\ Hamilton, ON, Canada.
}

Correspondence: Robert P. Dickson, Pulmonary and Critical Care Medicine, University of Michigan Health System, 6220 MSRB III/SPC 5642, 1150 W. Medical Center Dr., Ann Arbor, MI 48109-5642, USA. E-mail: rodicksolamed.umich.edu

@ERSpublications

Yet more evidence that the lung microbiome plays a role in idiopathic pulmonary fibrosis. But how close are we to proving causality? An editorial from Dickson, Harari and Kolb in the ERJ. http://bit.ly/ 2Pslow4

Cite this article as: Dickson RP, Harari S, Kolb M. Making the case for causality: what role do lung microbiota play in idiopathic pulmonary fibrosis?. Eur Respir J 2020; 55: 2000318 [https://doi.org/10.1183/ 13993003.00318-2020].

Though the notion of a "lung microbiome", i.e. a complex community of microbes inhabiting and influencing the lower respiratory tract, is barely a decade old [1], there is nothing new about the idea that the lungs represent a crucial interface between us and the microbial universe we inhabit. Louis Pasteur demonstrated the presence of viable microbes in air [2], and one of his contemporaries calculated that healthy adults inhale between 1500 and 14000 organisms each hour [3]. Numerous radiographic studies have repeatedly demonstrated the ubiquity of subclinical microaspiration [4-6], even in healthy, asymptomatic subjects. The surface area of the lungs is twice that of the gastrointestinal tract [7] and 30 times that of the skin [8], representing the body's largest interface with the outside environment. Thoughtful investigators of lung biology have long suspected that the mechanisms our lungs evolved to cope with this continuous microbial exposure (innate and adaptive immune defences, the establishment and resolution of fibrosis) play central roles in the pathogenesis of lung disease. As pulmonary biologist Galen Toews commonly said, long before the lung microbiome era: "All lung disease is infectious disease" (figure 1).

In the past decade, this intuition has been vindicated, especially relating to idiopathic pulmonary fibrosis (IPF). Since 2014, a cascade of studies (figure 2) have used culture-independent techniques to characterise and interrogate bacterial communities in the lungs of patients with IPF and animal models of the disease. The cumulative argument emerging from this field has upended old, simplistic notions regarding the supposed sterility of the lungs. The presence and significance of bacterial communities in the lungs of patients with IPF is now established and validated by numerous studies, robust across patient cohorts, laboratories, sequencing platforms and animal models. This evolution in our thinking of the role of 
FIGURE 1 Galen Toews (1945-2011). Long before the era of the lung microbiome, Dr Toews argued that "All lung disease is infectious disease."

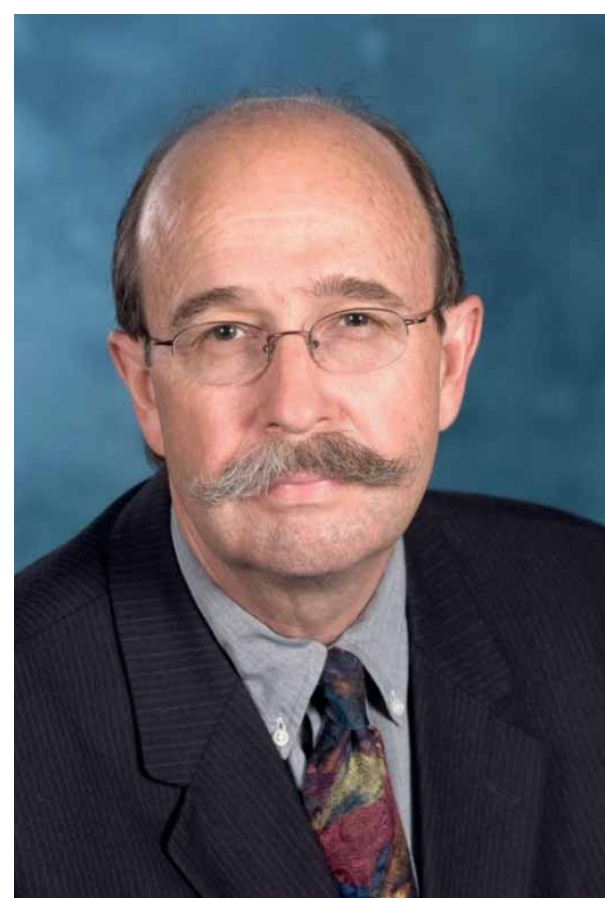

respiratory microbiology in IPF is already reflected in our clinical trials: whereas a decade ago we were studying immunosuppression in IPF [9], we are now instead trialling antibiotics [10].

Beyond the mere presence of a lung microbiome in IPF, the field has firmly established its correlative significance (figure 2): among patients with IPF, variation in lung microbiota correlates with variation in important aspects of the disease: disease status [11, 12], alveolar inflammation [13, 14], host genotype [11], and the systemic host response $[15,16]$. Further, several studies have independently established the prognostic significance of the lung microbiome in IPF: three separate analyses have demonstrated that variation in lung microbiota predict disease progression and mortality among patients with IPF $[11,13,17]$.

However provocative, these findings have not established the causal significance of the lung microbiome in IPF. A sceptic could fairly ask if lung microbiota in IPF are merely an indirect index of severity, reflecting some intrinsic, unmeasured feature of the disease (e.g. impaired mucociliary clearance in airways distorted by traction bronchiectasis). This is the central question facing the field [18]: is the lung microbiome merely an epiphenomenon in IPF, an innocent bystander to disease pathogenesis, or do altered lung microbiota directly participate in pathogenesis?

This core question was the rationale behind an important new study by INvERNizzi et al. [19] in this issue of the European Respiratory Journal. The authors studied a large cohort of patients with IPF who underwent both research bronchoscopy and quantitative interpretations of chest computed tomography (CT) scans. To characterise lung bacteria, the authors quantified total lung bacterial DNA burden, which has been the most consistent microbial predictor of clinical outcomes across respiratory conditions [11, 13, $20,21]$. In their analysis of CT imaging, they quantified the severity and extent of fibrosis, including key features such as traction bronchiectasis and honeycombing. They chose these radiographic findings given their known prognostic importance $[22,23]$ and because they provide objective insight into the anatomic distortion of IPF that may cause increased lung bacterial burden. The authors reasoned that if the prognostic significance of lung bacterial burden in IPF is merely an artefact of architectural destruction, then these two prognostic factors (microbiologic and radiographic) should be correlated with each other.

Instead, they found no relationship at all. Lung bacterial burden, while highly variable across patients, was unrelated to the radiographic severity of disease, whether considered collectively (e.g. fibrosis extent, or likelihood of usual interstitial pneumonia pattern) or in its specific features (e.g. traction bronchiectasis or honeycombing). Lung bacterial burden was similarly uncorrelated with physiological severity of disease (measured via forced vital capacity). These absences of association argue directly against the "innocent bystander" interpretation of the IPF lung microbiome literature. If lung bacterial burden is merely an indirect index of severity, why doesn't it correlate with our best available measurements of anatomic and physiologic severity? 


\section{Observation}

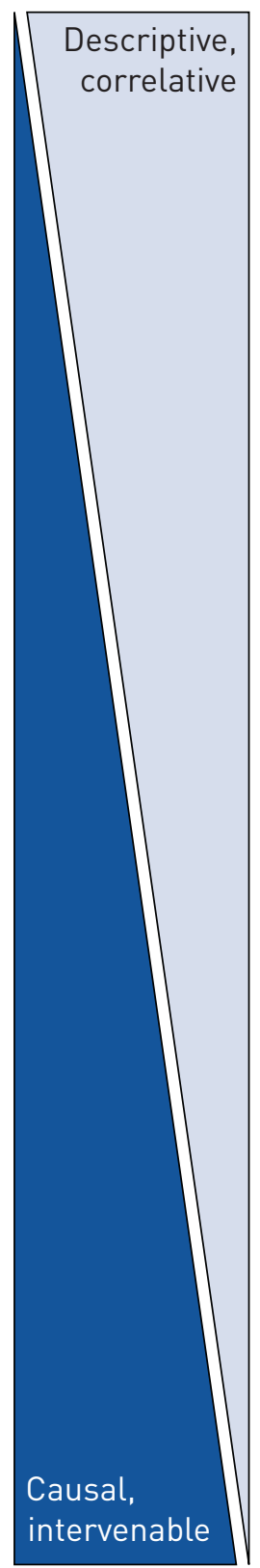

Lung microbiota are altered in patients with IPF relative to other conditions.

Lung microbiota are altered in animal models of IPF.

Lung microbiota correlate with disease severity in humans with IPF.

Lung bacterial community composition is correlated with radiographic features in IPF.

Lung bacterial burden is not correlated with radiographic features in IPF.

Lung microbiota are altered in acute exacerbations of IPF.

Lung microbiota are correlated with alveolar inflammation in IPF.

Lung microbiota are correlated with the systemic host response in IPF.

Lung microbiota are correlated with host genotype in IPF.

Lung microbiota are predictive of disease progression and mortality in IPF.

Manipulation of the lung microbiome is protective in animal models of IPF.

Lung bacterial community composition is altered by antibiotic therapy in animals.

Lung bacterial community composition is altered by antibiotic therapy in humans.

Lung bacterial burden is not altered by antibiotic therapy (azithromycin) in humans.

Manipulation of the lung microbiome is protective in humans with IPF.

\begin{tabular}{|c|c|c|c|c|c|c|c|}
\hline 2014 & 2015 & 2016 & 2017 & 2018 & 2019 & 2020 & Unknown \\
\hline \multirow[t]{8}{*}{$\begin{array}{c}\text { Molyneaux } \\
{[11]}\end{array}$} & & & & & $\begin{array}{l}\text { Tong } \\
\text { [12] }\end{array}$ & & \\
\hline & & & & $\begin{array}{c}\text { TAKAHASHI } \\
\text { [31] }\end{array}$ & $\begin{array}{c}\text { O'DWYER } \\
\text { [13] }\end{array}$ & & \\
\hline & & & & $\begin{array}{c}\text { TAKAHASHI } \\
\text { [31] }\end{array}$ & & & \\
\hline & & & & & $\begin{array}{c}\text { Dickson } \\
\text { [32] }\end{array}$ & & \\
\hline & & & & & & $\begin{array}{l}\text { INVERNIZZI } \\
\text { [19] }\end{array}$ & \\
\hline & & & $\begin{array}{c}\text { Molyneaux } \\
{[16]}\end{array}$ & & & & \\
\hline & & & $\begin{array}{l}\text { WANG } \\
{[14]}\end{array}$ & & $\begin{array}{c}\text { O'DWYER } \\
\text { [13] }\end{array}$ & & \\
\hline & & & $\begin{array}{c}\text { HUANG } \\
{[15]} \\
\text { MoLYNEAUX } \\
{[16]}\end{array}$ & & & & \\
\hline \multicolumn{8}{|l|}{$\begin{array}{c}\text { MolyneauX } \\
\text { [11] }\end{array}$} \\
\hline \multirow[t]{3}{*}{$\begin{array}{c}\text { Molyneaux } \\
{[11]} \\
\text { Han [17] }\end{array}$} & & & & & $\begin{array}{l}\text { O'DWYER } \\
\text { [13] }\end{array}$ & $\begin{array}{c}\text { INVERNIZZI } \\
\text { [19] }\end{array}$ & \\
\hline & & & & & $\begin{array}{c}\text { O'DWYER } \\
\text { [13] }\end{array}$ & & \\
\hline & & & & $\begin{array}{c}\text { Dickson } \\
\text { [24] }\end{array}$ & & & \\
\hline \multirow[t]{2}{*}{$\begin{array}{c}\text { SLATER } \\
\text { [25] } \\
\text { RoGERS } \\
{[26]} \\
\end{array}$} & & & $\begin{array}{l}\text { SEGAL } \\
{[27]}\end{array}$ & & & & \\
\hline & & & $\begin{array}{l}\text { SEGAL } \\
{[27]}\end{array}$ & & & $\begin{array}{l}\text { SPENCE } \\
{[28]}\end{array}$ & \\
\hline & & & & & & & \\
\hline
\end{tabular}

FIGURE 2 Building a causal argument for the role of the lung microbiome in idiopathic pulmonary fibrosis (IPF). In recent years, techniques of culture-independent microbiology have revolutionised our understanding of the significance of respiratory microbiota in IPF. The correlative significance of the lung microbiome in IPF is now inarguable: lung bacteria correlate with disease status, host genotype, and the alveolar and systemic host response. The current study by InvernIZZI et al. [19] definitively confirms the lung microbiome's prognostic significance: lung microbiota predict disease progression in IPF, independent of radiographic severity. Yet the causal significance of the lung microbiome in IPF, and its status as a therapeutic target, remains a crucial area of ongoing study, requiring both interventional human studies and translational animal modelling.

Yet in the same cohort, the authors again demonstrated the prognostic significance of lung bacterial burden in IPF. As in prior studies [11, 13], lung bacterial burden convincingly predicted clinical outcomes, defined here as all-cause mortality or spirometric progression at 12 months. This association remained robust to multivariable adjustment for key potential confounders that represent radiographic and physiologic severity of disease. While these findings do not definitively establish a causal role for lung microbiota in IPF pathogenesis, they do provide strong evidence that the lung microbiome's prognostic significance is not merely an artefact of disease severity. 
So what would it take to make the case for causality? We will need to demonstrate that manipulating the lung microbiome (therapeutically in humans, experimentally in animal models) alters the biology and clinical trajectory of IPF progression. This task is far easier to articulate than to accomplish, as it relies on several embedded claims: 1) the lung microbiome can be altered by interventions (e.g. antibiotics), 2) this manipulation influences the trajectory of IPF pathogenesis, and 3) the effects of these interventions are directly attributable to lung microbiome changes, and not off-target effects of therapy (e.g. the immunomodulating effects of macrolides, or the systemic effects of altered gut microbiota).

As shown in figure 2, the field has made some progress down this thorny path. Regarding the first embedded claim (the lung microbiome can be altered by interventions), it is now well-established that the community composition of lung bacteria is altered by antibiotics, both in animal models [24] and in human lung conditions other than IPF [25-27]. Yet it is unestablished if lung bacterial burden (the most consistently prognostic feature of the lung microbiome in $\operatorname{IPF}[11,13,19]$ and other conditions $[20,21])$ can be altered by antibiotics. In two studies of chronic macrolide therapy (in COPD patients [27] and lung transplant recipients [28]), chronic azithromycin had no effect on lung bacterial burden: the communities changed, but the total bacterial load was unaltered. If, as hoped, lung bacterial burden is to become a therapeutic target in IPF, we will first need to show that it can be modified, either via antibiotics or other interventions (e.g. fundoplication).

The second embedded claim, that manipulation of the lung microbiome influences IPF pathogenesis and progression, is supported by early animal and human observations. Germ-free mice (mice completely devoid of bacteria) are protected from mortality in the bleomycin model of pulmonary fibrosis [13]. In other murine models, the pathogenesis of pulmonary fibrosis is accelerated and augmented by pulmonary exposure to Streptococcus pneumoniae and its toxins [29], mirroring an association between lung enrichment with Streptococcus spp. and progression of IPF in human subjects [17]. A randomised human trial of co-trimoxazole in patients with IPF found benefit in all-cause mortality (though not in decline of lung function) [30]. An ongoing trial of antibiotics in IPF will provide more evidence regarding possible efficacy [10].

Yet none of these observations have addressed the third embedded claim: that the benefits of microbiome manipulation in IPF are not attributable to indirect, off-target effects of our interventions. To accomplish this, methodological advances are required both in our animal modelling and in our analysis of interventional human studies. In animal modelling, a central challenge is how to selectively alter lung microbiota without influencing gut microbiota, which inform the systemic immune response. In prospective human studies, we should be sampling and studying the lung microbiome both before and after randomised interventions: pre-treatment sampling to assess for heterogeneity of treatment effect (does variation in patients' baseline lung microbiota explain variation in their treatment response?), and post-treatment sampling for mediation analysis (does variation in patient's microbial response to therapy explain variation in their treatment response?). Finally, our current instruments for manipulating the microbiome (antibiotics and probiotics) are broad and nontargeted, analogous to using systemic corticosteroids to alter host immunity. A key challenge will be learning how to modify the lung microbiome with the same targeted precision with which we now modulate the host response (e.g. with monoclonal antibodies).

Despite these challenges, the future is bright. The revolution in our understanding of the microbiology of IPF has been thrilling, both for those who study IPF pathogenesis and for those who study respiratory microbiology. An entire kingdom of the tree of life, previously ignored in IPF research, is now a major focus of clinical and experimental study. We have much work left to do, but the current study by INVERNIZZi et al. [19] gives us reason to hope that we are on the path towards making a case for causality.

Conflict of interest: R.P. Dickson has nothing to disclose. S. Harari has nothing to disclose. M. Kolb reports grants and personal fees for consultancy and lectures from Roche and Boehringer Ingelheim, grants and personal fees for consultancy from GSK, Gilead and Prometic, grants from Actelion, Respivert, Alkermes and Pharmaxis, personal fees for consultancy from Genoa, Indalo and Third Pole, outside the submitted work.

\section{References}

1 Hilty M, Burke C, Pedro H, et al. Disordered microbial communities in asthmatic airways. PLoS One 2010; 5 : e8578.

2 Pasteur L. Expériences relatives aux générations dites spontanées. Comptes Rendus Hebdomadaires des Séances de l'Académie des Sciences D: Sciences Naturelles 1860; L: 303-307.

Thomson SC, Hewlett RT. The fate of micro-organisms in inspired air. Lancet 1896; 147: 86-87.

Quinn LH, Meyer OO. The relationship of sinusitis and bronchiectasis. Arch Otolaryngol 1929; 10: 152-165.

Huxley EJ, Viroslav J, Gray WR, et al. Pharyngeal aspiration in normal adults and patients with depressed consciousness. Am J Med 1978; 64: 564-568. 
6 Gleeson K, Eggli DF, Maxwell SL. Quantitative aspiration during sleep in normal subjects. Chest 1997; 111: 1266-1272.

Helander HF, Fandriks L. Surface area of the digestive tract - revisited. Scand J Gastroenterol 2014; 49: 681-689. Hasleton PS. The internal surface area of the adult human lung. J Anat 1972; 112: 391-400.

9 Raghu G, Anstrom KJ, King TE, Jr., et al. Prednisone, azathioprine, and N-acetylcysteine for pulmonary fibrosis. N Engl J Med 2012; 366: 1968-1977.

10 CleanUP IPF for the Pulmonary Trials Cooperative (CleanUp-IPF). https:/clinicaltrials.gov/ct2/show/ NCT02759120 Date last accessed: 7 February 2020. Date last updated: 17 September 2019.

11 Molyneaux PL, Cox MJ, Willis-Owen SA, et al. The role of bacteria in the pathogenesis and progression of idiopathic pulmonary fibrosis. Am J Respir Crit Care Med 2014; 190: 906-913.

12 Tong X, Su F, Xu X, et al. Alterations to the lung microbiome in idiopathic pulmonary fibrosis patients. Front Cell Infect Microbiol 2019; 9: 149.

13 O’Dwyer DN, Ashley SL, Gurczynski SJ, et al. Lung microbiota contribute to pulmonary inflammation and disease progression in pulmonary fibrosis. Am J Respir Crit Care Med 2019; 199: 1127-1138.

14 Wang J, Lesko M, Badri MH, et al. Lung microbiome and host immune tone in subjects with idiopathic pulmonary fibrosis treated with inhaled interferon-gamma. ERJ Open Res 2017; 3: 00008-2017.

15 Huang Y, Ma SF, Espindola MS, et al. Microbes are associated with host innate immune response in idiopathic pulmonary fibrosis. Am J Respir Crit Care Med 2017; 196: 208-219.

16 Molyneaux PL, Willis-Owen SAG, Cox MJ, et al. Host-microbial interactions in idiopathic pulmonary fibrosis. Am J Respir Crit Care Med 2017; 195: 1640-1650.

17 Han MK, Zhou Y, Murray S, et al. Lung microbiome and disease progression in idiopathic pulmonary fibrosis: an analysis of the COMET study. Lancet Respir Med 2014; 2: 548-556.

18 Segal LN, Molyneaux PL. The challenging road of moving from association to causation for microbiome research in idiopathic pulmonary fibrosis. Am J Respir Crit Care Med 2019; 199: 1054-1056.

19 Invernizzi R, Barnett J, Rawal B, et al. Bacterial burden in the lower airways predicts disease progression in idiopathic pulmonary fibrosis and is independent of radiological disease extent. Eur Respir J 2020; 55: 1901519.

20 Dickson RP, Schultz MJ, van der Poll T, et al. Lung microbiota predict clinical outcomes in critically ill patients. Am J Respir Crit Care Med 2020; 201: 555-563.

21 Sibila O, Laserna E, Shoemark A, et al. Airway bacterial load and inhaled antibiotic response in bronchiectasis. Am J Respir Crit Care Med 2019; 200: 33-41.

22 Sumikawa H, Johkoh T, Colby TV, et al. Computed tomography findings in pathological usual interstitial pneumonia: relationship to survival. Am J Respir Crit Care Med 2008; 177: 433-439.

23 Maldonado F, Moua T, Rajagopalan S, et al. Automated quantification of radiological patterns predicts survival in idiopathic pulmonary fibrosis. Eur Respir J 2014; 43: 204-212.

24 Dickson RP, Erb-Downward JR, Falkowski NR, et al. The lung microbiota of healthy mice are highly variable, cluster by environment, and reflect variation in baseline lung innate immunity. Am J Respir Crit Care Med 2018; 198: 497-508.

25 Slater M, Rivett DW, Williams L, et al. The impact of azithromycin therapy on the airway microbiota in asthma. Thorax 2014; 69: 673-674.

26 Rogers GB, Bruce KD, Martin ML, et al. The effect of long-term macrolide treatment on respiratory microbiota composition in non-cystic fibrosis bronchiectasis: an analysis from the randomised, double-blind, placebo-controlled BLESS trial. Lancet Respir Med 2014; 2: 988-996.

27 Segal LN, Clemente JC, Wu BG, et al. Randomised, double-blind, placebo-controlled trial with azithromycin selects for anti-inflammatory microbial metabolites in the emphysematous lung. Thorax 2017; 72: 13-22.

28 Spence CD, Vanaudenaerde B, Einarsson GG, et al. Influence of azithromycin and allograft rejection on the post-lung transplant microbiota. J Heart Lung Transplant 2020; 39: 176-183.

29 Knippenberg S, Ueberberg B, Maus R, et al. Streptococcus pneumoniae triggers progression of pulmonary fibrosis through pneumolysin. Thorax 2015; 70: 636-646.

30 Hammond M, Clark AB, Cahn AP, et al. The Efficacy and Mechanism Evaluation of Treating Idiopathic Pulmonary fibrosis with the Addition of Co-trimoxazole (EME-TIPAC): study protocol for a randomised controlled trial. Trials 2018; 19: 89.

31 Takahashi Y, Saito A, Chiba H. Impaired diversity of the lung microbiome predicts progression of idiopathic pulmonary fibrosis. Respir Res 2018; 19: 34.

32 Dickson RP, Huffnagle GB, Flaherty KR, et al. Radiographic honeycombing and altered lung microbiota in patients with idiopathic pulmonary fibrosis. Am J Respir Crit Care Med 2019; 200: 1544-1547. 